\title{
Squeezing Oil into Water under Pressure: Inverting the Hydrophobic Effect
}

Ciprian G. Pruteanu,* Victor Naden Robinson,* Narjes Ansari, Ali Hassanali, Sandro Scandolo, and John S. Loveday

Cite This: J. Phys. Chem. Lett. 2020, 11, 4826-4833

Read Online

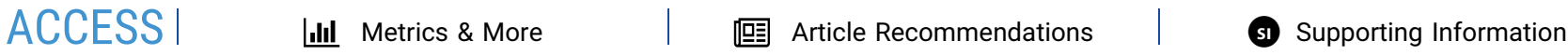

ABSTRACT: The molecular structure of dense homogeneous fluid watermethane mixtures has been determined for the first time using high-pressure neutron-scattering techniques at 1.7 and $2.2 \mathrm{GPa}$. A mixed state with a fully $\mathrm{H}$ bonded water network is revealed. The hydration shell of the methane molecules is, however, revealed to be pressure-dependent with an increase in the water coordination between 1.7 and $2.2 \mathrm{GPa}$. In parallel, $a b$ initio molecular dynamics simulations have been performed to provide insight into the microscopic mechanisms associated with the phenomenon of mixing. These calculations reproduce the observed phase change from phase separation to mixing with increasing pressure. The calculations also reproduce the experimentally observed structural properties. Unexpectedly, the simulations show mixing is accompanied by a subtle enhancement of the polarization of methane. Our results highlight the key role played by fine electronic effects on

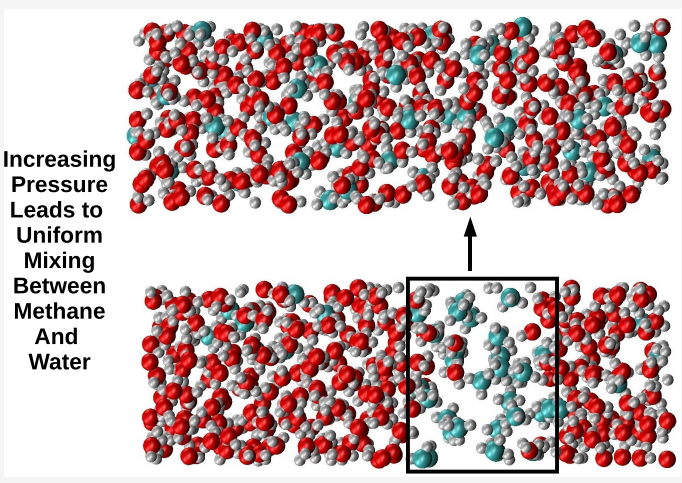
miscibility and the need to readjust our fundamental understanding of hydrophobicity to account for these.

$\mathrm{C}$ ommon experience tells us that oil and water are not miscible. ${ }^{1}$ The separation of oil and water is the cornerstone of the hydrophobic effect. ${ }^{2}$ Methane is the shortest hydrocarbon and is insoluble in water under ambient conditions ${ }^{1}\left(0.006 \mathrm{~mol} \%\right.$ solubility at $100{ }^{\circ} \mathrm{C}$ and $\left.10 \mathrm{MPa}\right)$. In this regard, the thermodynamics associated with the interaction of methane with water forms a classic textbook problem. Hydrophobic interactions are a fundamental and ubiquitous phenomenon because they underpin many crucial life-enabling processes such as protein folding ${ }^{3}$ and cellmembrane formation. ${ }^{4}$ Hydrophobicity also plays a central role in chemical engineering problems, for example, in the food industry. ${ }^{5}$

The evolution of hydrophobic interactions under different thermodynamic conditions is relevant to a wide range of science ranging from Earth and planetary sciences to biology and is consequently a longstanding active field of research. ${ }^{6-8}$ Methane-water mixtures are present at the bottom of oceans, where compression leads to the formation of solid methane hydrates; ${ }^{9}$ they are also major constituents of the middle layers of the ice giants Neptune and Uranus ${ }^{10}$ and icy satellites like Titan and Triton. ${ }^{11}$ Combinations of water, ammonia, and methane are predicted to be widely present in the recently observed exoplanets, which have most commonly been of Neptune-like proportions, ${ }^{12-14}$ and are likely to exist as either liquids or solids. ${ }^{15-18}$ A microscopic understanding of how extreme pressures modulate the solubility of methane-water mixtures is thus critical to develop realistic models of the interior of planets. ${ }^{19}$
Hydrophobic interactions are central in the underlying architecture of biological machinery. Over half a century ago, several different experiments showed the effects of pressure on biochemical processes such as protein folding and later on in chemical reactions. ${ }^{6}$ It is now well established, for example, that the phase diagram of proteins is very sensitive to pressure. $^{20-22}$ Specifically, single-chained proteins are known to denature at pressures of $\sim 100 \mathrm{MPa}$. Protein unfolding and denaturation have been generally linked to a pressure-induced weakening of hydrophobic interactions, ${ }^{23}$ but the molecular mechanisms associated with these phenomena remain poorly understood.

Methane generally shows a sublinear tendency of solubility increase with pressure in the range up to $\sim 1 \mathrm{GPa}$ so that the maximum solubility remains significantly below $1 \mathrm{~mol} \%{ }^{24,25}$ However, it was shown recently that starting at $1.3 \mathrm{GPa}$, the solubility of methane in water begins to dramatically increase and eventually saturates at a maximum of $44(3) \mathrm{mol} \%$ at 1.9 GPa and $373 \mathrm{~K}^{26}$ The microscopic origin of the increased solubility observed in these experiments is not clear. Chandler proposed that enhanced pressures would result in a disruption

Received: May 8, 2020

Accepted: June 4, 2020

Published: June 4, 2020 

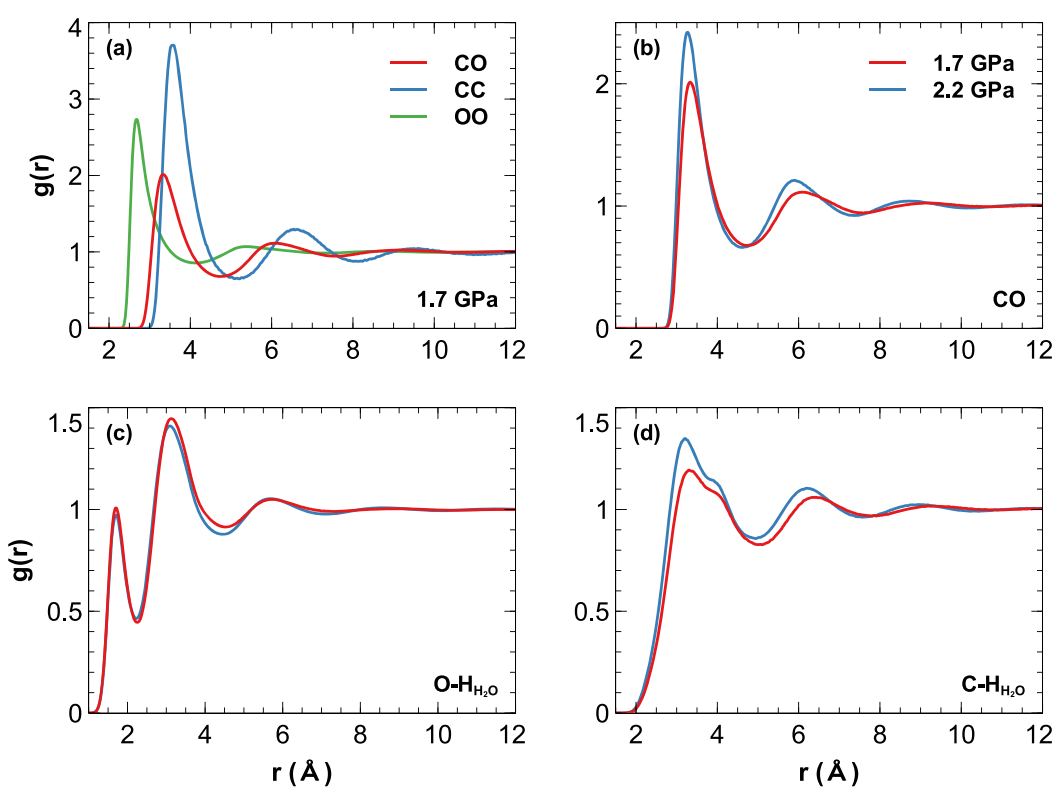

Figure 1. PDFs from EPSR. (a) Water-water, methane-methane, and methane-water for the sample at $1.7 \mathrm{GPa}$. (b) Methane-water (C-O) for both 1.7 and $2.2 \mathrm{GPa}$. (c) $\mathrm{O}-\mathrm{H}_{\mathrm{H}_{2} \mathrm{O}}$ for 1.7 and $2.2 \mathrm{GPa}$. (d) $\mathrm{C}-\mathrm{H}_{\mathrm{H}_{2} \mathrm{O}}$ for both 1.7 and $2.2 \mathrm{GPa}$. Changes in miscibility with pressure (such as $\mathrm{C}-$ $\mathrm{O})$ can be noticed; meanwhile, the $\mathrm{O}-\mathrm{O}$ remains largely unaffected. That is, the water-methane space is still much more compressible, whereas $\mathrm{O}-\mathrm{O}$ is at smaller separation than pure water at the same pressure.

of the hydrogen-bond network, leading to increased solubility. ${ }^{27}$ However, the extent to which hydrophobic molecules disrupt the hydrogen-bond network has been challenged by a combination of both simulations and experiment, which find that a fully $\mathrm{H}$-bonded network is maintained to high pressure. ${ }^{28,29}$ Moreover attempts to obtain a microscopic understanding failed because standard intermolecular potentials, with sufficiently large unit cells, do not give a mixed state. ${ }^{30}$

Here we report combined experimental and theoretical studies that reveal how the hydrophobic effect is inverted under pressure in methane-water mixtures. Our neutron scattering experiments reveal the local structure of the mixed fluid in the pressure range 1.7 to $2.2 \mathrm{GPa}$. $A b$ initio moleculardynamics simulations of the methane-water mixtures confirm the experimental observables and show an enhancement of interactions between the methane and water molecules due to a subtle increase in the methane dipole moment. These effects were not captured by standard classical potentials, which failed at reproducing pressure-induced enhanced solubility. ${ }^{30}$ The measured and calculated structure and topology of water show that methane mixing does not involve a significant change in the hydrogen-bond network as it adapts to the presence of methane.

\section{METHODS}

Experimental Details. Both measurements of the fluid structure were performed on the PEARL beamline at the ISIS Neutron Spallation Source, Rutherford-Appleton Laboratory, U.K. Samples of fully occupied methane hydrate I (MH-I, 5.75:1 water/methane content) were cryoloaded in a ParisEdinburgh high-volume press. ${ }^{31}$ A liquid-nitrogen-cooled TiZr gasket was filled with the sample and then placed in precooled anvils, and a 5 tonne sealing load was applied. The samples were compressed to the desired pressures and warmed to 413 $\mathrm{K}$ (where no crystalline content could be detected). Data were collected for $48 \mathrm{~h}$ for each run, followed by a $24 \mathrm{~h}$ collection of background and a $24 \mathrm{~h}$ collection of vanadium needed for normalization. Data analysis was performed using the EPSR (empirical potential software refinement) ${ }^{32}$ package having 1000 molecules simulation boxes with the same composition as the samples. The density of the fluid mixture was estimated using a linear combination of the known equations of state for fluid water ${ }^{33}$ and methane. ${ }^{34}$ After equilibration and fitting, the individual pair distribution functions were sampled over 10000 accumulations. For the initial setup of the simulation box, the molecules were defined using the TIP3P parameters for water (for $\mathrm{OH}$ bond lengths and dihedral angle) and the OPLS-AA parameters for methane, as described by Kaminski et al. ${ }^{35}$

Computational Details. The AIMD simulations were performed with the CP2K package using Quickstep. ${ }^{36}$ The wave function was expanded using both a TZVP Gaussian basis set and a plane-wave representation using a cutoff of 300 Ry. The Becke, Lee, Yang, and Parr (BLYP) exchangecorrelation, ${ }^{37,38}$ was used together with the Grimme D3 empirical corrections for the van der Waals interactions. ${ }^{39}$ Norm-conserving Goedecker-Teter-Hutter (GTH) pseudopotentials $^{40}$ were used for treating the core electrons. The AIMD simulations were integrated with a time step of $0.5 \mathrm{fs}$ within the NVT ensemble using the canonical sampling through velocity rescaling (CSVR) thermostat, ${ }^{41}$ thermostatting the system at $413 \mathrm{~K}$. Mixture boxes with 300 molecules had sizes at low pressure of $45.0 \times 15.0 \times 15.0 \AA$ corresponding to a density of $870 \mathrm{~kg} / \mathrm{cm}^{3}$ and a pressure of 0.2 $\mathrm{GPa}$, where high-pressure size refers to pressures of 1.2 and 2.3 GPa. (See the SI.) Initial conditions for mixed systems were created from the experimental $S(q)$ fitting and then expanded into a 311 orthorhombic box. Demixed systems were created from classical potential simulations, which favor this demixing. To reveal whether phase separation or mixing occurred, long simulations of up to 250 ps were performed.

Figure 1a shows the pair (or radial) distribution functions (PDFs) obtained from neutron scattering data. The totalscattering patterns (see the SI) obtained showed a few clearly 

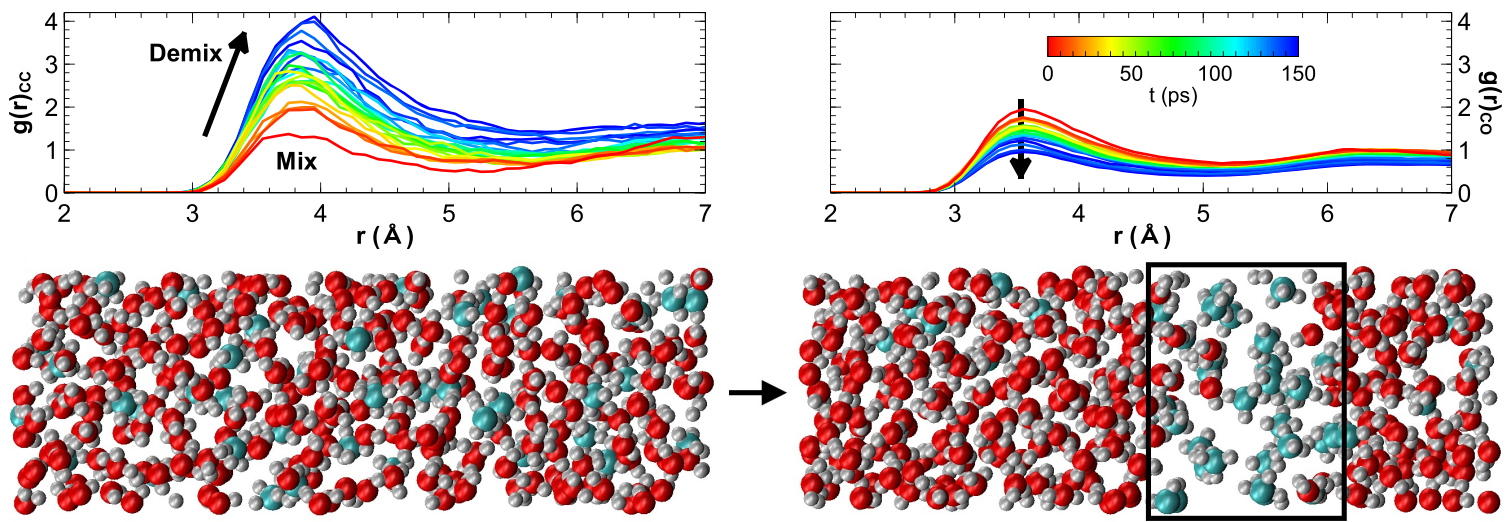

Figure 2. (Top) PDFs showing demixing at $0.2 \mathrm{GPa}$ as $\mathrm{CC}$ grows and $\mathrm{CO}$ reduces over time, forming the slab seen below. (Bottom) Initial and final $(150 \mathrm{ps})$ snapshots at $0.2 \mathrm{GPa}$, where the mixed system begins to phase-separate into a slab.
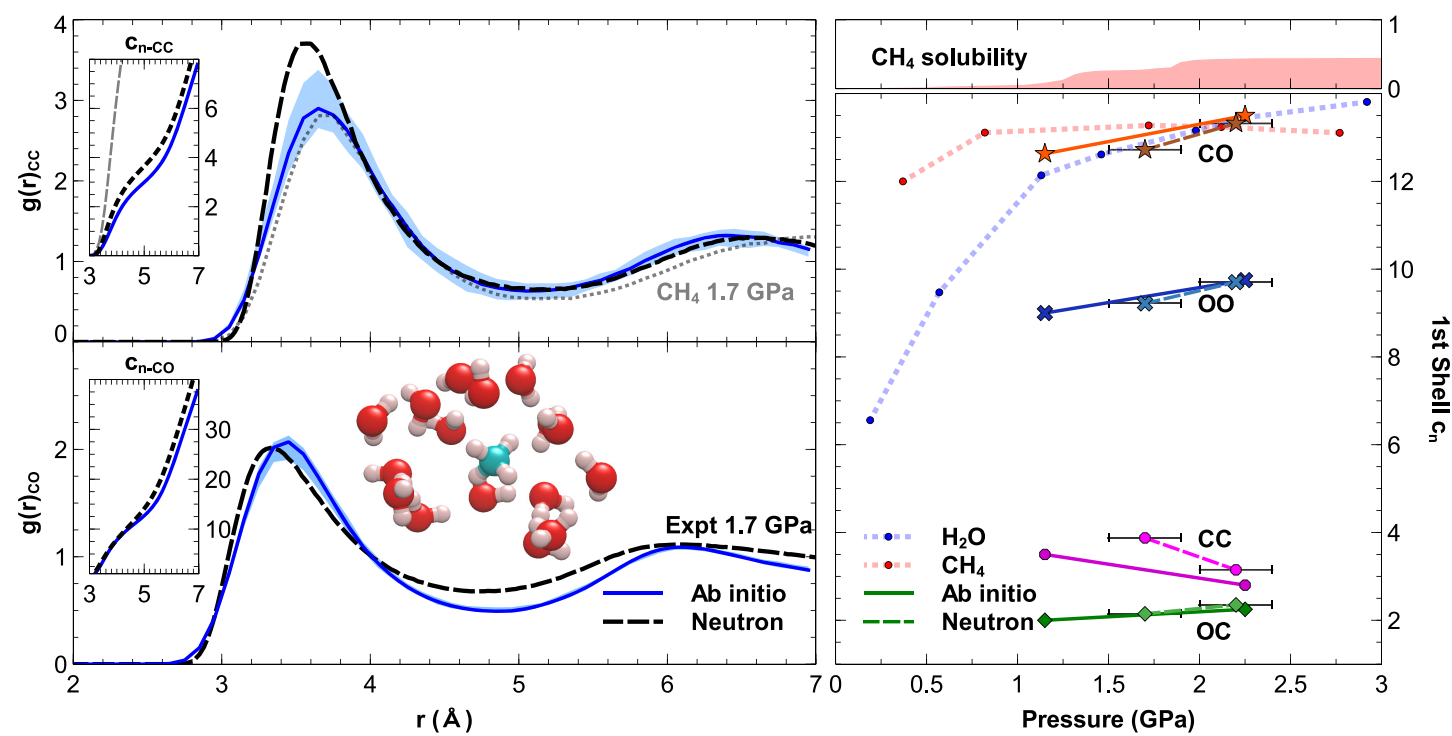

Figure 3. (Left) PDFs and inset running coordination numbers $\left(c_{n}\right)$ from AIMD (blue line, $1.2 \mathrm{GPa}$ ) compared with experimental results (dashed black line, $1.7 \mathrm{GPa}$ ) and those of pure phases from AIMD (dotted gray lines). A fluctuation confidence window is included, indicated by a blue shaded region. (Right) All $c_{n}$ values from integrating up to the first minima of PDFs for neutron data (dashed lines) and $a b$ initio data (solid lines). Top panel shows the maximal solubility as a function of pressure, as reported by Pruteanu et al. ${ }^{26}$ Pure systems are included for reference, and symbols match the type of atom pair. Whereas there are few data points, there is strong overlap between experiment and simulation, which are likely probing the very same liquid.

localized broad peaks with no sign of heterogeneity, ruling out the possibility of an emulsion. Molecular-level homogeneous mixing is supported by the further analysis: High-quality fits did not require methane or water clustering or cluster-size rescaling, as commonly encountered in emulsions. ${ }^{42}$ The extracted PDFs are all smooth and monotonically decaying, as expected for simple fluids. ${ }^{43}$ By contrast, more complex systems that present bonding and local ordering or clustering tend to yield significantly more feature-rich distribution functions, such as $\mathrm{SiO}_{2}$ melts. ${ }^{44}$ The total scattering data showed an increase in $\mathrm{CH}_{4}-\mathrm{H}_{2} \mathrm{O}$ coordination with increasing pressure (Figure $1 \mathrm{~b}$ ), and a simultaneous decrease in $\mathrm{CH}_{4}-$ $\mathrm{CH}_{4}$ coordination is seen in Figure 3. This is accompanied by very little change in the structure of water, which tends to maintain its normal coordination and $\mathrm{H}$-bonding structure (Figure 1c). Conversely, the decreased methane-methane coordination is accompanied by increased water coordination. (See Figure $1 \mathrm{~b}, \mathrm{~d}$.) Both the $\mathrm{C}-\mathrm{O}$ and $\mathrm{C}-\mathrm{H}_{\mathrm{H}_{2} \mathrm{O}}$ partial $g(r)$ values show clear increases in the height of the first peak at 2.2
GPa. Integration of these curves out to the first minimum shows an increase in water coordination of $\sim 0.6$ molecules. Furthermore, the first coordination shell sharpens so that the coordination out to $4.38 \AA$ increases by almost two water molecules.

Using EPSR's built-in capabilities to calculate spatial correlation functions, especially spatial distribution functions and dipole moment orientations, we found that water's dipole moment points away from the methane molecules (see the SI), similar to the situation reported at lower pressure by Koh et $\mathrm{al}^{45}$ during hydrate formation. Moreover, Buchanan et al. ${ }^{46}$ found that at $180 \mathrm{bar}$ and $18{ }^{\circ} \mathrm{C}$, the presence of dissolved methane in water causes a compression of the second shell of water, similar to but smaller than the effect of high pressure on pure water. We notice a significantly more collapsed second shell due to the combination of both higher pressure and over an order of magnitude more methane being present in the mixture ( $14.8 \mathrm{~mol} \%$ in our case, $0.3 \mathrm{~mol} \%$ in Buchanan's). The authors also noted that there are $16 \pm 1$ water molecules 

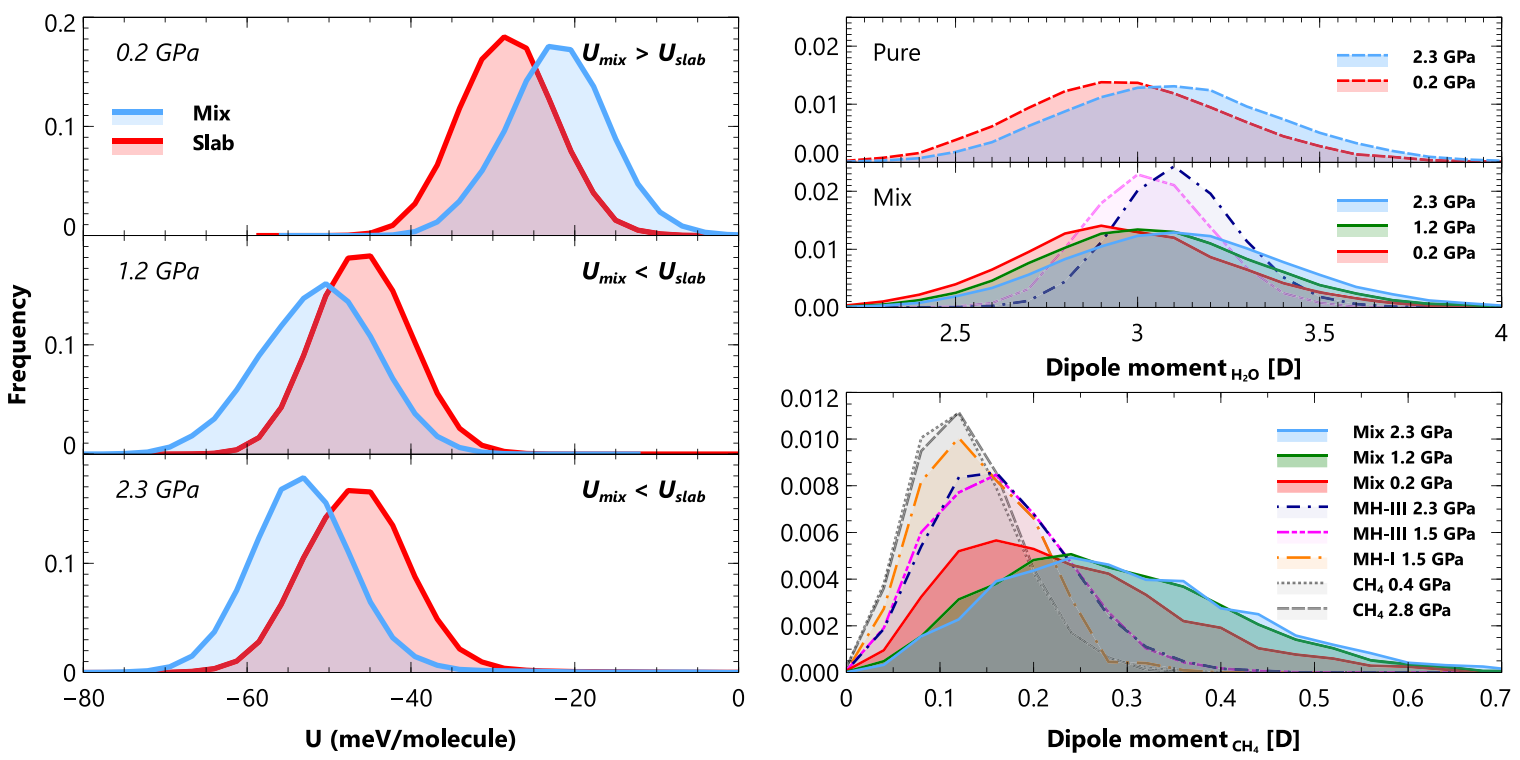

Figure 4. (Left) Distribution of internal energy, $U$, plotted for the different systems in mixed or demixed states. With increasing pressure, the internal energy shows that the mixed system becomes more stable. (Right) Distribution of dipole moments for methane and water molecules. Increasing pressure shows that the water dipole does not significantly change, although the mixed system has a greater dipole moment due to the shorter $\mathrm{O}-\mathrm{O}$ distance for the same pressure as the pure system. For methane, the mixed system causes a subtle enhancement of dipole moment due to the presence of dense water molecules, far more so than in the solid hydrates (I,III) shown at $300 \mathrm{~K}$.

coordinating a given methane molecule, whereas we find slightly fewer than and greater than 13 water molecules for 1.7 and $2.2 \mathrm{GPa}$, respectively. The number of neighboring water molecules is expected to decrease in a solution having a significantly higher methane content despite also being at much higher pressure (which naturally tends to increase the number of neighbors at a given fixed distance).

To provide more insights into the molecular origins of the pressure-induced mixing, we performed a series of $a b$ initio molecular dynamics simulations. Ab initio simulations, while more accurate than classical simulations because they explicitly include the electronic degrees of freedom, are computationally more intensive and thus require prudent choice of system sizes and simulation times. A series of different initial conditions were constructed to study the mixing/demixing at $0.2,1.2$, and 2.3 GPa. Specifically, we began with two limiting cases: a mixed configuration obtained from the EPSR refinement at 1.7 $\mathrm{GPa}$ and a slab-like geometry corresponding to an initial demixed state. (See the Methods.)

Figure 2 highlights some of the key initial findings of our simulations that confirm the experimental observations. Initial and final snapshots obtained from a 130 ps trajectory at 0.2 GPa clearly illustrate that the system irreversibly demixes to form a slab-like geometry. This is also quantified in the timedependent PDFs shown at the top of Figure 2 reporting an increase in the methane coordination and a decrease in the solvent exposure of the methane molecules over time.

This phenomenon is distinct from the interfacial mixing previously observed at ambient pressure ${ }^{47-49}$ and shows that our simulations are able to mix and demix the system on $a b$ initio time scales.

Initial and final configurations that were obtained from the simulations at 1.2 GPa starting from a slab geometry are shown in the SI. Interestingly, over a time scale of $30-50 \mathrm{ps,}$ we observe water molecules penetrating into the slab, which results in methane molecules breaking away from the cluster. The time scales associated with the mixing are very slow, and the simulations (200 ps at $1.2 \mathrm{GPa}$ ) are not long enough to capture the kinetics associated with this process required to generate fully mixed configurations. After 200 ps at $1.2 \mathrm{GPa}$ and $250 \mathrm{ps}$ at $2.3 \mathrm{GPa}$, respectively, the mixing process is evident yet slower than the demixing at $0.2 \mathrm{GPa}$.

To assess the quality of the mixed methane-water simulations, we show in Figure 3 the pair distribution functions and associated coordination numbers for the mixture at 1.2 GPa. The differences observed between the experimental and simulation PDFs are largely due to the different pressures of 1.2 and $1.7 \mathrm{GPa}$ and are reflected more in the $g(r)_{\mathrm{CC}}$. These differences can also be attributed to the simulation's sensitivity to equilibration, differences in the mixing concentration (14 vs $14.8 \%)$, the possibility of having small emulsions in the sample, the EPSR $\mathrm{CH}$ bond length and the OPLSS-AA methane potential, and the $a b$ initio simulations lacking a perfect physical description of interactions. Better agreement could be made if the initial conditions were closer to the $g(r)$ distributions that EPSR converged upon. (See the SI.) The fact that the pure $\mathrm{CH}_{4} g(r)_{\mathrm{CC}}$ is not hugely different from that of the mixture implies that $\mathrm{CH}_{4}-\mathrm{CH}_{4}$ distances are similar but with much lower $\mathrm{CC} c_{n}$ in the mixture.

The pressure evolution of the coordination numbers and a comparison with the pure phases of water and methane, respectively, are shown in Figure 3. Consistent with the PDFs previously reported, there is a clear increase with pressure in the number of oxygen atoms within the first shell $\left(c_{n}\right)$ of a given methane molecule, accompanied by a similar decrease in the number of neighboring methane molecules. The structure of solvated water molecules (water molecules neighboring other water molecules) also increases but remains lower than that of pure water due to the excluded-volume effect owing to the presence of methane. Computed spatial distribution functions from AIMD reinforce this (see the SI) and are in good agreement with those calculated by EPSR. One can also notice that at $\sim 2 \mathrm{GPa}$, the number of water molecules within the first shell of a methane molecule in the mixture equals that 

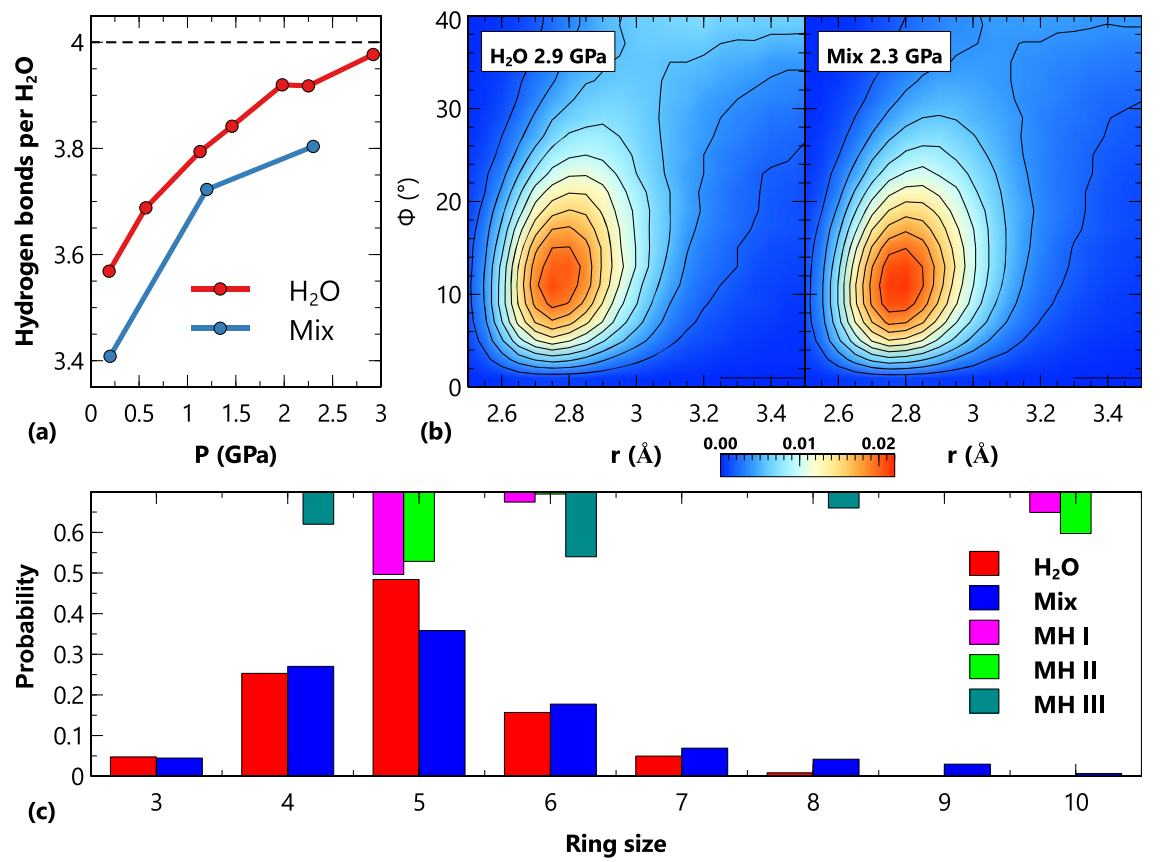

Figure 5. (a) Water hydrogen bonds (cutoff at $3.5 \AA$ and $35^{\circ}$ ) per water molecule and (b) $2 \mathrm{D}$ probability distribution of closest hydrogen bonds for pure water at $2.9 \mathrm{GPa}$ and the mix at $2.3 \mathrm{GPa}$. (c) Ring statistics at 1.2 (mixture) and $1.1 \mathrm{GPa}$ (water) with ticks plotted at the top representing closed rings found in methane hydrate $(\mathrm{MH})$ phases I-III.

of all neighboring molecules in the pure phase. The effect of solvating methane we observe here is akin to previous simulations by Hummer and coworkers, which examined the potential of mean force using classical potentials between two methane molecules in water as a function of pressure. ${ }^{23}$ An increase in pressure appears to destabilize the contact minimum between two methane molecules and stabilize the solvent-separated state.

The combined experimental and simulation results up to this point show that an increase in pressure leads to suppression of the hydrophobic effect. To understand better the microscopic origin of this phenomenon, we next examine the thermodynamic signatures associated with mixing. It is beyond the scope of the current calculations to compute the free energies of mixing/demixing. However, mixing is always entropically favored, and, in this particular case, the entropy of mixing is found to be close to that of an ideal random mixture, at both 1.2 and 2.3 GPa (calculated using the same method as in Pruteanu et al.; ${ }^{30}$ see the SI). We will therefore focus on the evolution of the internal energy on mixing. In the left panel of Figure 4, the internal energies of mixed and unmixed simulations are shown at $0.2,1.2$, and $2.3 \mathrm{GPa}$. At $0.2 \mathrm{GPa}$, $U$ was calculated before the system starts to phase separate. Interestingly, at both 1.2 and $2.3 \mathrm{GPa}$, the mixed simulation of methane and water is lower in energy, by $\sim 10 \mathrm{meV} /$ molecule. At $0.2 \mathrm{GPa}$, where the methane molecules cluster together, the slab conditions are lower in energy compared with the mixed state. The stabilization of the methane-water interactions at high pressure thus correspond to $\sim 12 \mathrm{meV} /$ molecule, roughly $5 \%$ of the cohesive energy for a water molecule dimer. This suggests that internal energy is strongly influencing the mixing driving force and importantly turns over from not favoring to favoring mixing with pressure.

Previously, we stated that previously performed classical molecular dynamics simulations failed, even qualitatively, to reproduce the trends observed in the experiments. ${ }^{30}$ One possible origin of this failure is that the increase in pressure leads to subtle changes in the electronic properties of both methane and water, which cannot be captured by the TIP3P, TIP4P, SPCE, and OPLS-AA potentials, for example, in the molecular polarization. Previous theoretical studies have also suggested the importance of methane polarization in methane hydrates. ${ }^{50}$ To assess the role of polarization in our simulations, we examined the molecular dipole distributions of pure, mixed, and slab-like systems. The dipole moment was calculated using maximally localized Wannier functions (WCs). ${ }^{51}$ The total molecular dipole is given by $\boldsymbol{D}_{\text {tot }}=$ $-\sum_{i} 2 e r_{i}+\sum_{I} Q_{I} R_{I}$ where $R_{I}$ is the position of the ion center, $\boldsymbol{r}_{i}$ is the position of the Wannier center, and $Q_{I}$ is the ionic charge.

The dipole moments are shown for water and methane molecules in the top and bottom panels, respectively. Overall, the increase in pressure results in an increase in the dipole moment of water by $0.13 \mathrm{D}$ at $2.3 \mathrm{GPa}$ : the dipole moments in pure water at ambient pressure and $2.3 \mathrm{GPa}$ are $2.97 \pm 0.08$ and $3.10 \pm 0.09 \mathrm{D}$, respectively. (The uncertainties are estimated from the variances.) For methane in the mixture, the change is quite remarkable. At just $1.2 \mathrm{GPa}$, the dipole moment increases to roughly $0.3 \mathrm{D}$ and remains almost the same at $2.3 \mathrm{GPa}$. For reference, methane molecules in the pure phase are nonpolar and are characterized by a very small and pressure-independent dipole moment and fluctuations (calculated here to be $0.1 \mathrm{D}$ ). For the $0.2 \mathrm{GPa}$ mixed system, the snapshots were taken from the first part of the simulation before the system had demixed. The increased dipole moments of the methane molecules we observe are consistent with previous $a b$ initio simulations that were performed at much higher temperatures and pressures, ${ }^{17}$ yet here they occur at much lower pressures and temperatures.

It is well appreciated the inclusion of dispersion interactions is critical for reproducing both the structural and dynamical properties of aqueous systems with density functional theory 
(DFT) ${ }^{52}$ Although we have included dispersion interactions using the empirical Grimme correction, we wanted to assess the sensitivity of some of our results to the choice of using more accurate nonlocal dispersion functionals. For this reason, we performed simulations using both $\mathrm{DRSLL}^{53}$ and $\mathrm{rVV}^{54}$ functionals for the methane-water mixtures at $1.2 \mathrm{GPa}$ and for 20 ps. The dipole moment distributions are consistently reproduced by all of these functionals.

Methane in the gas phase would, due to symmetry, have no permanent dipole. The nonzero methane dipole seen in our simulations arises from fluctuation-induced symmetry breaking. This is a feature that appears to be enhanced at high pressure relative to ambient conditions. At high pressure, the presence of a large fluctuating dipole has already been observed in previous first-principles simulations. ${ }^{17} \mathrm{We}$ find that the magnitude of the fluctuating dipole of methane is not short-lived (see SI Figure 34), which shows the dipole moment for a single methane molecule as a function of time. The magnitude of the dipole fluctuates between 0.05 and $0.7 \mathrm{D}$ on the time scales of picoseconds, whereas the average of the magnitude of the dipole moments of all $\mathrm{CH}_{4}$ molecules is roughly constant at $0.3 \mathrm{D}$. As expected, the average of each component of the dipole vector of methane is zero; however, the fluctuations away from zero are roughly twice as large in the mixture at $1.2 \mathrm{GPa}$ compared with the pure methane system. It is these strong fluctuations of the vector from zero that boost the magnitude of the dipole moment for methane.

The enhanced dipole moment of methane under pressure does not imply that it has a permanent dipole moment, but a finite dipole arises from enhanced fluctuations of the three components of the dipole vector. In SI Figure 32, we observe that all three components of the dipole are well approximated by Gaussian distributions, with the same spread implying no symmetry breaking. However, under pressure, the enhanced fluctuations of all three components leads to an increase in methane's dipole moment, which is consistent with previous studies. $^{17}$

The increase in interactions between the methane molecules and water has a rather subtle effect on the water hydrogenbond network. We first examined the number of hydrogen bonds formed between water molecules both in pure water up to $3 \mathrm{GPa}$ and in the methane-water mixtures, which is shown in Figure 5. Hydrogen bonds were determined by using a geometrical criterion developed by Luzar and Chandler. ${ }^{55}$ Interestingly, we observe that the water hydrogen-bond network retains its integrity in the mixture. We find that the mixed system contains $97 \%$ of $\mathrm{H}$ bonds found in the pure water system at both 1.2 and $2.3 \mathrm{GPa}$. Although there are slightly fewer hydrogen bonds, they tend to be slightly stronger than in pure water: The bond angle and bond distance distributions are slightly smaller, with greater weight at smaller angles and distances, in the mixture. (See the SI.) Our results are thus fully consistent with some recent experiments and simulations by Grdadolnik et al. ${ }^{29}$ that found a tendency for pure hydrophobic solutes to strengthen and enhance hydrogen bonding rather than weaken it. It is likely that increased methane content $(>14 \%)$ reduces the local dipole field of the water molecules, and this may limit the solubility of methane to the observed value of $44 \%$ at $3 \mathrm{GPa}$.

To examine the structural effects of methane mixing at high pressure on the topology of the hydrogen-bond network, we examined the distribution of closed rings made up of hydrogen-bonded water molecules in the different systems.
Ring distributions have been in used in several previous studies to provide insight into the changes on the hydrogen-bond network of water in the presence of solutes. ${ }^{56-59}$ The ring statistics are shown in Figure $5 \mathrm{c}$ for three different systems: the mixed methane-water system, the demixed slab system, and pure water at $1.5 \mathrm{GPa}$. We observe that all three systems are dominated by the presence of five-membered rings. However, the number of five-membered rings is highest for the pure water system and lowest for the methane mixture. Furthermore, the presence of water molecules interspersed by methane also results in an increase in the number of larger rings, as seen in the fatter tail of rings with six members or greater.

The presence of 4/5/6/8-membered rings has also been observed in low-pressure methane clathrates, ${ }^{60,61}$ indicated in Figure 5 . The mixture exhibits a greater number of rings, with a greater difference for six-membered, consistent with MH-III (stable above $1.9 \mathrm{GPa}$ ), implying a similar clathrate-like structure in the mixed liquid but with greater flexibility for defects in the ring networks. Comparing liquid dipoles with $\mathrm{MH}-\mathrm{III}$, the mixture has greater fluctuations in both water and methane molecules. The methane dipole in the mixed liquid is stronger than that in MH-III, partly due to the difference in methane content $(0.14$ vs 0.33$)$ and due to the geometry restrictions of $\mathrm{MH}$-III where methane molecules exist as pairs. However, methane dipoles in MH-I at $1.5 \mathrm{GPa}$ reveal a distribution closer to that of pure methane, even though the MH-I methane content is $14 \%$, as in the mixed liquid. In the solid hydrates, increasing pressure shows a tendency to increase the methane dipole moment, but we find this effect to be much stronger in the liquid.

The combined experimental and $a b$ initio modeling studies have revealed the structure of the pressure-mixed methanewater fluid. It has a fully hydrogen-bonded water network without significant broken hydrogen bonds and increasing water to methane coordination with increasing pressure and a reduction in the width of the hydration shell. The modeling provides insight into what is changing at the onset of mixing. At a macroscopic level, mixing appears to be driven by a change with pressure in the enthalpy of mixing rather than a change in the entropy of mixing. Mixing is also accompanied by a change in the ring connectivity of the water network with a trend to larger rings than pure water.

Our results begin to understand the importance of electronic polarization on hydrophobic effects in soft-matter systems. Protein-water interactions, for example, will clearly be affected by subtle changes in the polarizability, in particular, under pressure, and play an important role in tuning the phase diagram of protein stability. This opens up interesting challenges for the development of empirical force fields for biological systems under extreme pressures.

\section{ASSOCIATED CONTENT}

\section{SI Supporting Information}

The Supporting Information is available free of charge at https://pubs.acs.org/doi/10.1021/acs.jpclett.0c01410.

Simulation snapshots; polarization and Wannier centers; methane hydrate dipoles; vibrational density of states; table of simulation details; pressure temperature phase diagram; equation of state and pressure determination; structure factors; angular distribution functions; entropy of mixing; orientation dynamics; methane hydrate pair 
distribution functions; fluid mixture pair distribution functions; spatial density functions and dipole moment orientations; and dispersion, $\mathrm{XC}$, and dipoles (PDF)

\section{AUTHOR INFORMATION}

\section{Corresponding Authors}

Ciprian G. Pruteanu - Department of Physics and Astronomy, University College London, London WC1E 6BT, United Kingdom; 이이.org/0000-0001-6586-4115; Email: cip.pruteanu@ucl.ac.uk

Victor Naden Robinson - The "Abdus Salam" International Centre for Theoretical Physics, I-34151 Trieste, Italy;

Email: victornadenrobinson@gmail.com

\section{Authors}

Narjes Ansari - The "Abdus Salam" International Centre for Theoretical Physics, I-34151 Trieste, Italy; 이이이.org/00000003-2017-8431

Ali Hassanali - The "Abdus Salam" International Centre for Theoretical Physics, I-34151 Trieste, Italy

Sandro Scandolo - The "Abdus Salam" International Centre for Theoretical Physics, I-34151 Trieste, Italy

John S. Loveday - SUPA, School of Physics and Astronomy and Centre for Science at Extreme Conditions, The University of Edinburgh, Edinburgh EH9 3JZ, United Kingdom;

(1) orcid.org/0000-0003-3985-9982

Complete contact information is available at:

https://pubs.acs.org/10.1021/acs.jpclett.0c01410

\section{Notes}

The authors declare no competing financial interest.

\section{ACKNOWLEDGMENTS}

This work was supported by the Engineering and Physical Sciences Research Council (EPSRC) through a Centre for Doctoral Training studentship and a Doctoral Prize Fellowship for C.G.P. We thank ISIS Neutron Spallation Source, U.K. for the award of beamtimes (RB1820413 and RB1700058) and Dr. Craig Bull for assistance during the experiments; UCL Research Computing Services for CPU time on Grace; and CINECA for computing resources on Marconi.

\section{REFERENCES}

(1) Atkins, P.; De Paula, J.; Keeler, J. Atkins' Physical Chemistry; Oxford University Press, 2018.

(2) Chandler, D. Hydrophobicity: Two Faces of Water. Nature 2002, 417, 491.

(3) Zhou, R.; Huang, X.; Margulis, C. J.; Berne, B. J. Hydrophobic Collapse in Multidomain Protein Folding. Science 2004, 305, 16051609.

(4) Tanford, C. The Hydrophobic Effect and the Organization of Living Matter. Science 1978, 200, 1012-1018.

(5) Di Bonaventura, G.; Piccolomini, R.; Paludi, D.; D'orio, V.; Vergara, A.; Conter, M.; Ianieri, A. Influence of Temperature on Biofilm Formation by Listeria Monocytogenes on Various Foodcontact Surfaces: Relationship with Motility and Cell Surface Hydrophobicity. J. Appl. Microbiol. 2008, 104, 1552-1561.

(6) Daniel, I.; Oger, P.; Winter, R. Origins of Life and Biochemistry Under High-Pressure Conditions. Chem. Soc. Rev. 2006, 35, 858-875.

(7) Ancilotto, F.; Chiarotti, G. L.; Scandolo, S.; Tosatti, E. Dissociation of Methane into Hydrocarbons at Extreme (Planetary) Pressure and Temperature. Science 1997, 275, 1288-1290.

(8) Schaack, S.; Ranieri, U.; Depondt, P.; Gaal, R.; Kuhs, W. F.; Gillet, P.; Finocchi, F.; Bove, L. E. Observation of Methane Filled
Hexagonal Ice Stable up to 150 GPa. Proc. Natl. Acad. Sci. U. S. A. 2019, 116, 16204-16209.

(9) Hoffmann, R. Old Gas, New Gas. Am. Sci. 2006, 94, 16-18.

(10) Bergstralh, J. T.; Miner, E. D.; Matthews, M. S. Uranus; University of Arizona Press, 1991.

(11) Grasset, O.; Sotin, C.; Deschamps, F. On the Internal Structure and Dynamics of Titan. Planet. Space Sci. 2000, 48, 617-636.

(12) Hubbard, W. B.; MacFarlane, J. J. Structure and Evolution of Uranus and Neptune. J. Geophys. Res. 1980, 85, 225-234.

(13) Rauer, H.; Catala, C.; Aerts, C.; Appourchaux, T.; Benz, W.; Brandeker, A.; Christensen-Dalsgaard, J.; Deleuil, M.; Gizon, L.; Goupil, M. J.; et al. The PLATO 2.0 Mission. Exp. Astron. 2014, 38, 249-330.

(14) Noack, L.; Snellen, I.; Rauer, H. Water in Extrasolar Planets and Implications for Habitability. Space Sci. Rev. 2017, 212, 877-898.

(15) Naden Robinson, V.; Wang, Y.; Ma, Y.; Hermann, A. Stabilization of Ammonia-rich Hydrate Inside Icy Planets. Proc. Natl. Acad. Sci. U. S. A. 2017, 114, 9003-9008.

(16) Naden Robinson, V.; Marqués, M.; Wang, Y.; Ma, Y.; Hermann, A. Novel Phases in Ammonia-Water Mixtures under Pressure. J. Chem. Phys. 2018, 149, 234501.

(17) Lee, M.-S.; Scandolo, S. Mixtures of Planetary Ices at Extreme Conditions. Nat. Commun. 2011, 2, 185.

(18) Bethkenhagen, M.; Cebulla, D.; Redmer, R.; Hamel, S. Superionic Phases of the 1:1 Water-Ammonia Mixture. J. Phys. Chem. A 2015, 119, 10582-10588.

(19) Marley, M. S.; Fortney, J. J. Encyclopedia of the Solar System; Elsevier, 2014; pp 743-758.

(20) Doster, W.; Friedrich, J. Protein Folding Handbook; John Wiley \& Sons, Ltd., 2008; Chapter 5, pp 99-126.

(21) Smeller, L. Pressure-Temperature Phase Diagrams of Biomolecules. Biochim. Biophys. Acta, Protein Struct. Mol. Enzymol. 2002, 1595, 11-29.

(22) Panick, G.; Vidugiris, G. J. A.; Malessa, R.; Rapp, G.; Winter, R.; Royer, C. A. Exploring the Temperature Pressure Phase Diagram of Staphylococcal Nuclease. Biochemistry 1999, 38, 4157-4164.

(23) Hummer, G.; Garde, S.; Garcia, A. E.; Paulaitis, M. E.; Pratt, L. R. The Pressure Dependence of Hydrophobic Interactions is Consistent with the Observed Pressure Denaturation of Proteins. Proc. Natl. Acad. Sci. U. S. A. 1998, 95, 1552-1555.

(24) Duan, Z.; Mao, S. A. Thermodynamic Model for Calculating Methane Solubility, Density and Gas Phase Composition of Methanebearing Aqueous Fluids from 273 to $523 \mathrm{~K}$ and from 1 to 2000 bar. Geochim. Cosmochim. Acta 2006, 70, 3369-3386.

(25) Sun, R.; Duan, Z. An Accurate Model to Predict the Thermodynamic Stability of Methane Hydrate and Methane Solubility in Marine Environments. Chem. Geol. 2007, 244, 248-262.

(26) Pruteanu, C. G.; Ackland, G. J.; Poon, W. C.; Loveday, J. S. When Immiscible Becomes Miscible-Methane in Water at High Pressures. Sci. Adv. 2017, 3, No. e1700240.

(27) Chandler, D. Interfaces and the Driving Force of Hydrophobic Assembly. Nature 2005, 437, 640.

(28) Strässle, T.; Saitta, A.; Le Godec, Y.; Hamel, G.; Klotz, S.; Loveday, J.; Nelmes, R. Structure of Dense Liquid Water by Neutron Scattering to $6.5 \mathrm{GPa}$ and $670 \mathrm{~K}$. Phys. Rev. Lett. 2006, 96, 067801.

(29) Grdadolnik, J.; Merzel, F.; Avbelj, F. Origin of Hydrophobicity and Enhanced Water Hydrogen Bond Strength near Purely Hydrophobic Solutes. Proc. Natl. Acad. Sci. U. S. A. 2017, 114, 322-327.

(30) Pruteanu, C. G.; Marenduzzo, D.; Loveday, J. S. PressureInduced Miscibility Increase of $\mathrm{CH}_{4}$ in $\mathrm{H}_{2} \mathrm{O}$ : A Computational Study Using Classical Potentials. J. Phys. Chem. B 2019, 123, 8091.

(31) Besson, J.; Nelmes, R. New Developments in NeutronScattering Methods under High Pressure with the Paris-Edinburgh Cells. Phys. B 1995, 213, 31-36.

(32) Soper, A. Tests of the Empirical Potential Structure Refinement Method and a New Method of Application to Neutron Diffraction Data on Water. Mol. Phys. 2001, 99, 1503-1516. 
(33) Li, M.; Li, F.; Gao, W.; Ma, C.; Huang, L.; Zhou, Q.; Cui, Q. Brillouin Scattering Study of Liquid Methane under High Pressures and High Temperatures. J. Chem. Phys. 2010, 133, 044503.

(34) Abramson, E. H.; Brown, J. M. Equation of State of Water based on Speeds of Sound Measured in the Diamond-Anvil Cell. Geochim. Cosmochim. Acta 2004, 68, 1827-1835.

(35) Kaminski, G.; Duffy, E. M.; Matsui, T.; Jorgensen, W. L. Free Energies of Hydration and Pure Liquid Properties of Hydrocarbons from the OPLS All-Atom Model. J. Phys. Chem. 1994, 98, 1307713082 .

(36) VandeVondele, J.; Krack, M.; Mohamed, F.; Parrinello, M.; Chassaing, T.; Hutter, J. Quickstep: Fast and Accurate Density Functional Calculations using a Mixed Gaussian and Plane Waves Approach. Comput. Phys. Commun. 2005, 167, 103-128.

(37) Becke, A. D. Density-Functional Exchange-Energy Approximation with Correct Asymptotic Behavior. Phys. Rev. A: At., Mol., Opt. Phys. 1988, 38, 3098.

(38) Lee, C.; Yang, W.; Parr, R. G. Development of the ColleSalvetti Correlation-Energy Formula into a Functional of the Electron Density. Phys. Rev. B: Condens. Matter Mater. Phys. 1988, 37, 785.

(39) Grimme, S.; Antony, J.; Ehrlich, S.; Krieg, H. A Consistent and Accurate $\mathrm{Ab}$ Initio Parametrization of Density Functional Dispersion Correction (DFT-D) for the 94 Elements H-Pu. J. Chem. Phys. 2010, 132, 154104.

(40) Krack, M. Pseudopotentials for $\mathrm{H}$ to $\mathrm{Kr}$ Optimized for Gradient-Corrected Exchange-Correlation Functionals. Theor. Chem. Acc. 2005, 114, 145-152.

(41) Bussi, G.; Donadio, D.; Parrinello, M. Canonical Sampling through Velocity Rescaling. J. Chem. Phys. 2007, 126, 014101.

(42) Hansen, P. H.; Rödner, S.; Bergström, L. Structural Characterization of Dense Colloidal Films Using a Modified Pair Distribution Function and Delaunay Triangulation. Langmuir 2001, 17, 4867-4875.

(43) Yarnell, J.; Katz, M.; Wenzel, R. G.; Koenig, S. Structure Factor and Radial Distribution Function for Liquid Argon at $85 \mathrm{~K}$. Phys. Rev. A: At., Mol., Opt. Phys. 1973, 7, 2130.

(44) Prescher, C.; Prakapenka, V. B.; Stefanski, J.; Jahn, S.; Skinner, L. B.; Wang, Y. Beyond Sixfold Coordinated $\mathrm{Si}$ in $\mathrm{SiO}_{2}$ Glass at Ultrahigh Pressures. Proc. Natl. Acad. Sci. U. S. A. 2017, 114, 1004110046 .

(45) Koh, C. A.; Wisbey, R. P.; Wu, X.; Westacott, R. E.; Soper, A. K. Water Ordering around Methane during Hydrate Formation. J. Chem. Phys. 2000, 113, 6390-6397.

(46) Buchanan, P.; Aldiwan, N.; Soper, A.; Creek, J.; Koh, C. Decreased Structure on Dissolving Methane in Water. Chem. Phys. Lett. 2005, 415, 89-93.

(47) Sakamaki, R.; Sum, A. K.; Narumi, T.; Ohmura, R.; Yasuoka, K. Thermodynamic Properties of Methane/water Interface Predicted by Molecular Dynamics Simulations. J. Chem. Phys. 2011, 134, 144702.

(48) English, N. J.; Lauricella, M.; Meloni, S. Massively Parallel Molecular Dynamics Simulation of Formation of Clathrate-hydrate Precursors at Planar Water-methane Onterfaces: Insights into Heterogeneous Nucleation. J. Chem. Phys. 2014, 140, 204714.

(49) Sæthre, B. S.; van der Spoel, D.; Hoffmann, A. C. Free Energy of Separation of Structure II Clathrate Hydrate in Water and a Light Oil. J. Phys. Chem. B 2012, 116, 5933-5940.

(50) Jiang, H.; Jordan, K. D.; Taylor, C. E. Molecular Dynamics Simulations of Methane Hydrate Using Polarizable Force Fields. J. Phys. Chem. B 2007, 111, 6486-6492.

(51) Marzari, N.; Vanderbilt, D. Maximally Localized Generalized Wannier Functions for Composite Energy Bands. Phys. Rev. B: Condens. Matter Mater. Phys. 1997, 56, 12847.

(52) Santra, B.; Klimeš, J. c. v.; Alfe, D.; Tkatchenko, A.; Slater, B.; Michaelides, A.; Car, R.; Scheffler, M. Hydrogen Bonds and van der Waals Forces in Ice at Ambient and High Pressures. Phys. Rev. Lett. 2011, 107, 185701 .

(53) Dion, M.; Rydberg, H.; Schröder, E.; Langreth, D. C.; Lundqvist, B. I. Van der Waals Density Functional for General Geometries. Phys. Rev. Lett. 2004, 92, 246401.
(54) Klimeš, J.; Bowler, D. R.; Michaelides, A. Chemical Accuracy for the Van der Waals Density Functional. J. Phys.: Condens. Matter 2010, 22, 022201.

(55) Luzar, A.; Chandler, D. Hydrogen-bond Kinetics in Liquid Water. Nature 1996, 379, 55.

(56) Head-Gordon, T. Is Water Structure Around Hydrophobic Groups Clathrate-like? Proc. Natl. Acad. Sci. U. S. A. 1995, 92, 83088312 .

(57) Santra, B., Jr.; DiStasio, R. A.; Martelli, F.; Car, R. Local Structure Analysis in Ab Initio Liquid Water. Mol. Phys. 2015, 113, 2829-2841.

(58) Ansari, N.; Dandekar, R.; Caravati, S.; Sosso, G.; Hassanali, A. High and Low Density Patches in Simulated Liquid Water. J. Chem. Phys. 2018, 149, 204507.

(59) Ansari, N.; Laio, A.; Hassanali, A. Spontaneously Forming Dendritic Voids in Liquid Water Can Host Small Polymers. J. Phys. Chem. Lett. 2019, 10, 5585-5591.

(60) Loveday, J.; Nelmes, R.; Guthrie, M.; Klug, D.; Tse, J. Transition from Cage Clathrate to Filled Ice: the Structure of Methane Hydrate III. Phys. Rev. Lett. 2001, 87, 215501.

(61) Ranieri, U.; Koza, M. M.; Kuhs, W. F.; Klotz, S.; Falenty, A.; Gillet, P.; Bove, L. E. Fast Methane Diffusion at the Interface of Two Clathrate Structures. Nat. Commun. 2017, 8, 1076. 